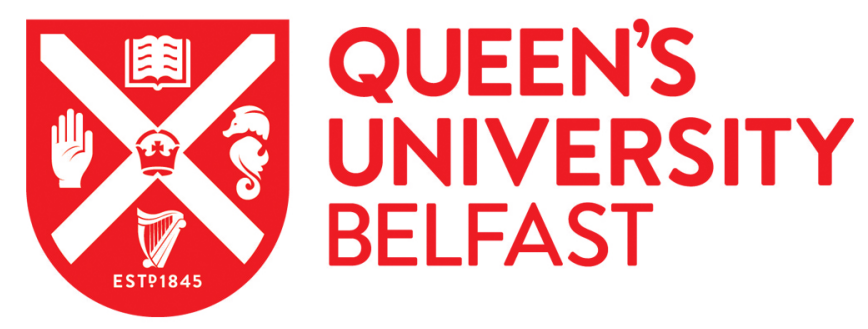

\title{
Using the fluorescent properties of STO-609 as a tool to assist structure-function analyses of recombinant CaMKK2
}

Gerner, L., Munack, S., Temmerman, K., Lawrence-Dörner, A-M., Besir, H., Wilmans, M., Jensen, J. K., Thiede, B., Mills, I. G., \& Morth, J. P. (2016). Using the fluorescent properties of STO-609 as a tool to assist structurefunction analyses of recombinant CaMKK2. Biochemical and Biophysical Research Communications, 476(2), 102-107. https://doi.org/10.1016/j.bbrc.2016.05.045

Published in:

Biochemical and Biophysical Research Communications

Document Version:

Peer reviewed version

Queen's University Belfast - Research Portal:

Link to publication record in Queen's University Belfast Research Portal

\section{Publisher rights}

Copyright Elsevier 2016

This manuscript is distributed under a Creative Commons Attribution-NonCommercial-NoDerivs License

(https://creativecommons.org/licenses/by-nc-nd/4.0/), which permits distribution and reproduction for non-commercial purposes, provided the author and source are cited.

\section{General rights}

Copyright for the publications made accessible via the Queen's University Belfast Research Portal is retained by the author(s) and / or other copyright owners and it is a condition of accessing these publications that users recognise and abide by the legal requirements associated with these rights.

Take down policy

The Research Portal is Queen's institutional repository that provides access to Queen's research output. Every effort has been made to ensure that content in the Research Portal does not infringe any person's rights, or applicable UK laws. If you discover content in the Research Portal that you believe breaches copyright or violates any law, please contact openaccess@qub.ac.uk. 


\section{Accepted Manuscript}

Using the fluorescent properties of STO-609 as a tool to assist structure-function analyses of recombinant CaMKK2

Lisa Gerner, Steffi Munack, Koen Temmerman, Ann-Marie Lawrence-Dörner, Hüseyin Besir, Matthias Wilmans, Jan Kristian Jensen, Bernd Thiede, Ian G. Mills, Jens Preben Morth

PII: S0006-291X(16)30737-9

DOI: 10.1016/j.bbrc.2016.05.045

Reference: $\quad$ YBBRC 35795

To appear in: Biochemical and Biophysical Research Communications

Received Date: 3 May 2016

Accepted Date: 9 May 2016

Please cite this article as: L. Gerner, S. Munack, K. Temmerman, A.-M. Lawrence-Dörner, H. Besir, M. Wilmans, J.K. Jensen, B. Thiede, I.G. Mills, J.P. Morth, Using the fluorescent properties of STO-609 as a tool to assist structure-function analyses of recombinant CaMKK2, Biochemical and Biophysical Research Communications (2016), doi: 10.1016/j.bbrc.2016.05.045.

This is a PDF file of an unedited manuscript that has been accepted for publication. As a service to our customers we are providing this early version of the manuscript. The manuscript will undergo copyediting, typesetting, and review of the resulting proof before it is published in its final form. Please note that during the production process errors may be discovered which could affect the content, and all legal disclaimers that apply to the journal pertain. 


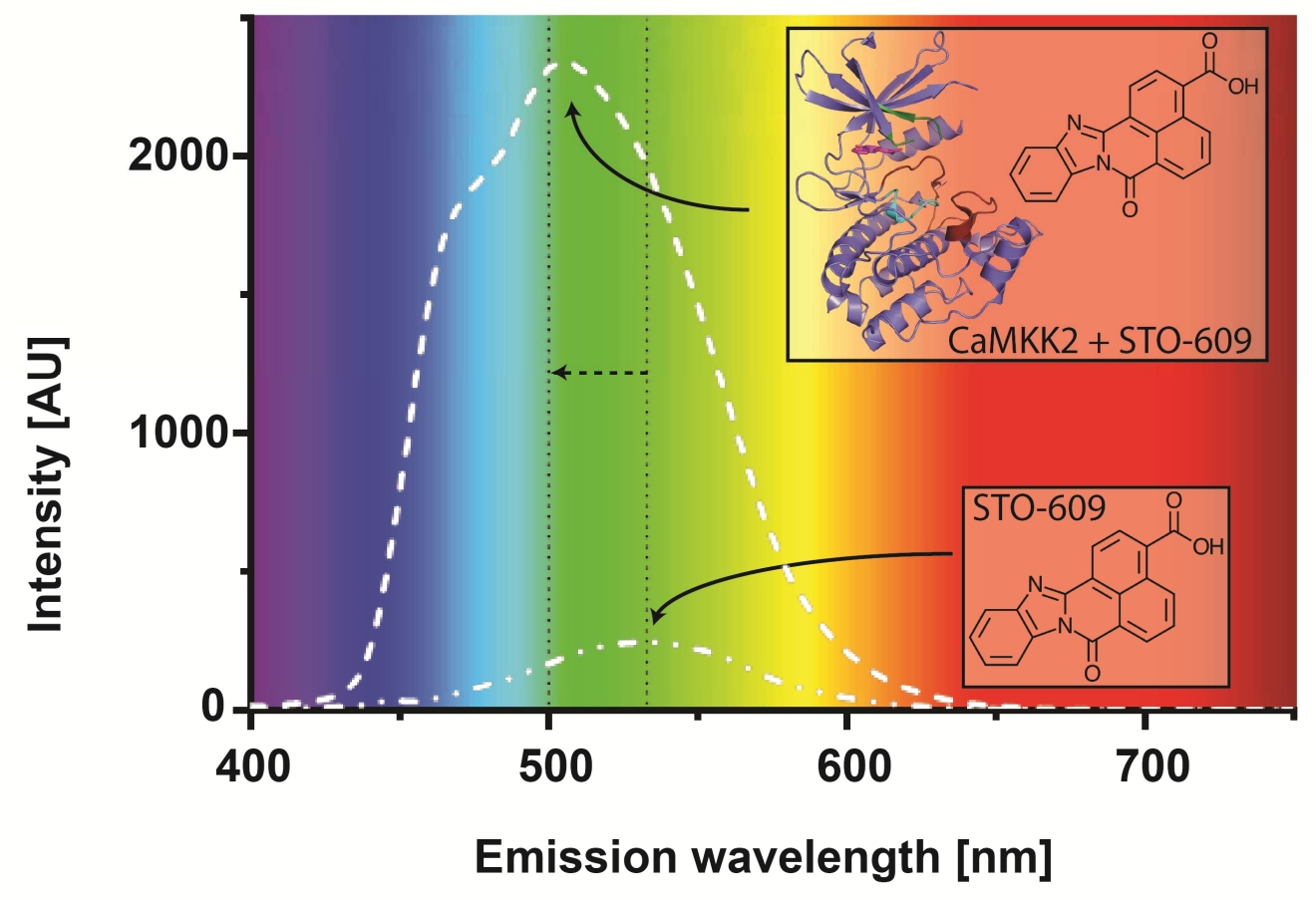




\section{Using the fluorescent properties of STO-609 as a tool to assist structure-function analyses of recombinant CaMKK2}

\section{Author names and affiliations}

Lisa Gerner ${ }^{a}$, Steffi Munack ${ }^{a}$, Koen Temmerman ${ }^{b, c}$, Ann-Marie Lawrence-Dörner ${ }^{c, 1}$, Hüseyin Besir $^{c}$, Matthias Wilmans ${ }^{b}$, Jan Kristian Jensen ${ }^{d}$, Bernd Thiede ${ }^{e, 2}$, lan G. Mills ${ }^{a, 3,4 *}$, Jens Preben Morth ${ }^{\mathrm{a}, 5 *}$

*Joint senior authors

\section{Addresses}

${ }^{a}$ Centre for Molecular Medicine Norway (NCMM), Nordic EMBL Partnership, Forskningsparken, University of Oslo and Oslo University Hospitals, 0349, Oslo, Norway; ${ }^{\mathrm{b}}$ European Molecular Biology Laboratory Hamburg, Notkestrasse 85, 22603 Hamburg, Germany; ${ }^{\circ}$ European Molecular Biology Laboratory Heidelberg, Meyerhofstrasse 1, 69117 Heidelberg, Germany; ${ }^{d}$ Department of Molecular Biology and Genetics, Aarhus University, 8000 Aarhus, Denmark; ${ }^{\mathrm{e}}$ Department of Biosciences, University of Oslo, Norway

${ }^{1}$ Institute of Biochemistry, WWU Münster, Wilhelm-Klemm-Straße 2, 48149 Münster, Germany; ${ }^{2}$ Biotechnology Centre of Oslo, University of Oslo, Norway; ${ }^{3}$ Department of Molecular Oncology, Institute for Cancer Research, Oslo University Hospitals, Norway; ${ }^{4}$ Movember/Prostate Cancer UK Centre of Excellence for Prostate Cancer Research, Centre for Cancer Research and Cell Biology (CCRCB), Queen's University Belfast, UK; ${ }^{5}$ Institute for Experimental Medical Research, Oslo University Hospital, Norway

\section{Corresponding authors}


*To whom correspondence should be addressed: ian.mills@ncmm.uio.no; i.mills@qub.ac.uk and j.p.morth@ncmm.uio.no

\section{Abbreviations}

AMPK, adenosine monophosphate-regulated kinase; CaM, calmodulin; CaMK, calcium/calmodulin-dependent kinase; CaMKK2, calcium/calmodulin-dependent kinase kinase 2; CaMKK2:CaM, CaMKK2-CaM complex

\section{Abstract}

Calcium/calmodulin-dependent kinase kinase 2 (CaMKK2) has been implicated in the regulation of metabolic activity in cancer and immune cells, and affects whole-body metabolism by regulating ghrelin-signalling in the hypothalamus. This has led to efforts to develop specific CaMKK2 inhibitors, and STO-609 is the standardly used CaMKK2 inhibitor to date. We have developed a novel fluorescence-based assay by exploiting the intrinsic fluorescence properties of STO-609. Here, we report an in vitro binding constant of $\mathrm{K}_{\mathrm{D}}$ 17 nM between STO-609 and purified CaMKK2 or CaMKK2:Calmodulin complex. Whereas high concentrations of ATP were able to displace STO-609 from the kinase, GTP was unable to achieve this confirming the specificity of this association. Recent structural studies on the kinase domain of CaMKK2 had implicated a number of amino acids involved in the binding of STO-609. Our fluorescent assay enabled us to confirm that Phe ${ }^{267}$ is critically important for this association since mutation of this residue to a glycine abolished the binding of STO-609. An ATP replacement assay, as well as the mutation of the 'gatekeeper' amino acid Phe ${ }^{267}$ Gly, confirmed the specificity of the assay and once more confirmed the strong binding of STO-609 to the kinase. In further characterising the purified kinase and kinasecalmodulin complex we identified a number of phosphorylation sites some of which corroborated previously reported CaMKK2 phosphorylation and some of which, particularly in the activation segment, were novel phosphorylation events. 
In conclusion, the intrinsic fluorescent properties of STO-609 provide a great opportunity to utilise this drug to label the ATP-binding pocket and probe the impact of mutations and other regulatory modifications and interactions on the pocket. It is however clear that the number of phosphorylation sites on CaMKK2 will pose a challenge in studying the impact of phosphorylation on the pocket unless the field can develop approaches to control the spectrum of modifications that occur during recombinant protein expression in $E$. coli.

\section{Keywords}

CaMKK2, STO-609, phosphorylation, fluorescent probe, drug screening, kinome 


\section{Introduction}

Calcium/calmodulin-dependent kinase kinase 1 and 2 (CaMKK1 and CaMKK2) has been identified as kinases capable of phosphorylating calcium/calmodulin-dependent kinases 1 and 4 (CaMK1 and CaMK4) and the adenosine monophosphate-regulated kinase (AMPK) [14]. CaMKK2 activity is itself also highly regulated both by phosphorylation and by binding to calcium-calmodulin. Of the multiple phosphorylation sites within CaMKK2 some have been shown in cell lines to be dependent on the activity of cyclin-dependent kinase 5 (CDK5) and glycogen synthase kinase 3 (GSK3) [5]. A number of autophosphorylation sites have also been identified within CaMKK2. Phosphorylation can occur both in mammalian cell lines and during recombinant expression of the kinase in bacteria. The relationship between these regulatory modifications and interactions remains to be fully defined. CaMKK2 is predominantly expressed in the brain and central nervous system and in immune cells [6-8]. In the brain and the central nervous system it promotes glycolytic metabolism in the neurons as well as regulating whole-body energy metabolism by functioning downstream of ghrelin in the hypothalamus $[9,10]$. In immune cells it has been reported to sustain macrophage proliferation in response to inflammation and immunological insults [8]. CaMKK2 was also recently reported to be overexpressed in prostate cancers under the control of a transcription factor, the androgen receptor, and to enhance glycolysis and anabolic metabolism to support tumour development [11].

These physiological associations have led to considerable interest over the last decade in the development of CaMKK2 inhibitors and the most specific example to date, called STO609 (PubChem CID: 3467590), is known to be a selective ATP-competitive CaMKK inhibitor $[12,13]$. STO-609 is a naphthoyl fused benzimidazole cell-permeable compound reported to have a low nanomolar $\mathrm{IC}_{50}$ value as determined by in vitro kinase assays against CaMKKs [12]. However, it also inhibits a number of other kinases including one of its primary 
substrates, AMPK, at a $1 \mu \mathrm{M}$ concentration in the same assays and is often used at 10 $20 \mu \mathrm{M}$ when applied to cell-lines [14]. Consequently in order to progress CaMKK2 inhibitors into a clinical setting additional drug development is required. Despite the intense interest in the function of this kinase only two structures have yet been determined; the inhibitor-kinase domain complex [13] and a peptide-derived from the kinase in complex with calmodulin (CaM) [15]. Consequently in order to accelerate the development of new CaMKK2 inhibitors and to obtain further mechanistic understanding of this important kinase, more structural and biophysical data are needed.

Here we report the development of an in vitro assay that assesses the binding properties of STO-609 by exploiting the intrinsic fluorescent properties. We performed this assay using recombinant human full-length CaMKK2, CaMKK2 $\mathrm{F}^{267} \mathrm{G}$ mutant, and CaMKK2 in complex with calmodulin (CaMKK2:CaM), for which we report the complete expression (E. coli) and purification protocol in the attached Date in Brief report, section 2. The novel fluorescent in vitro assay has allowed us to characterise the relationship between STO-609 and CaMKK2, both in the presence and absence of calcium and CaM. Furthermore, we have established that recombinant expressed CaMKK2 constructs were highly phosphorylated and that this had a pronounced effect on the binding measurements performed with STO-609 on CaMKK2.

These findings highlight the importance for careful assessment of the protein composition/quality in kinase drug screenings. Moreover, this assay offers a drug screen for future drug development initiatives that is not kinase activity-dependent, and we would propose using this assay in an STO-609 displacement screen with new candidate inhibitors. This assay would be applicable both for CaMKK2 and other kinases inhibited by STO-609 [1]. 


\section{Material and Methods}

Unless otherwise specified, all chemicals and reagents were purchased from Sigma-Aldrich at BioUltra reagent grade.

\section{Fluorescence Spectroscopy}

All fluorescent data was collected using a FP8500 spectrofluorometer (Jasco, Germany) with monochrometer slit widths set at $5 \mathrm{~nm}$ at $24^{\circ} \mathrm{C}$. Mea surements were taken in $1.5 \mathrm{ml}$ protein buffer $\left(30 \mathrm{mM}\right.$ Hepes $\mathrm{pH} 7.2,120 \mathrm{mM} \mathrm{NaCl}, 1 \mathrm{mM} \mathrm{CaCl}_{2}$ ) in a $1 \times 1 \mathrm{~cm}$ quartz cuvette (Hellma, Inc.) including STO-609 or the sample protein at the stated concentrations, respectively. Fluorescence titrations were performed by incremental additions $(1 \mu \mathrm{l})$ of concentrated CaMKK2 $(20 \mu \mathrm{M})$, STO-609 $(5 \mu \mathrm{M})$, ATP $(50 \mu \mathrm{M}$ and $50 \mathrm{mM})$ or EGTA pH 8.0 $(0.5 \mathrm{M})$, respectively, under continuous mixing at $600 \mathrm{rpm}$. For all titration experiments we have used an excitation wavelength of $400 \mathrm{~nm}$ and collected emission data at $500 \mathrm{~nm}$ at $0.5 \mathrm{~nm}$ intervals to quantitate effects of bound CaMKK2 to STO-609. All experiments were repeated a minimum of three times. The fluorescence contribution of STO-609 titrated into buffer in the absence of protein was subtracted from all experimental data.

\section{Fluorescent data analysis for determining affinity constant $K_{D}$}

The volume corrected change in fluorescence $(\Delta F)$ after each addition of STO-609 was estimated according to equation 1.

$F_{i v}=\left(\frac{V_{0}}{V_{0}+v_{i}}\right) * F_{i}$

With $\mathrm{V}_{0}$ as the initial sample volume, $v_{i}$ is volume of titrant added in ith increments throughout the experiment. $F_{i}$ is the fluorescence signal observed and $F_{i v}$ volume adjusted signal measurement were recorded at an excitation wavelength of $400 \mathrm{~nm}$ and an emission wavelength of $500 \mathrm{~nm}$. 
$F_{i v}=F_{o}+\left(F_{\text {final }}-F_{0}\right)\left(\frac{\left(N *\left[\text { CaMKK2] }+[\text { STO }]+K_{D}\right)\right.}{2 * N *[\text { CaMKK2] }}-\frac{\left.\sqrt{\left(N *[\text { CaMKK2 }]+[\text { STO }]+K_{D}\right)^{2}-4[\text { CaMKK2][STO }]}\right)}{2 * N *[\text { CaMKK2] }}\right)$

The obtained values for $F_{i v}$ were plotted as a function of the STO-609 concentration. Spectra were corrected by subtraction of background buffer scans, but were not smoothed. We used the data analysis program GraphPad Prism version 6.00 for Mac (GraphPad Software, www.graphpad.com) to analyse the generated data points to obtain a value for the $K_{D}$ assuming only a single binding site $(\mathrm{N}=1)$. [CaMKK2] is the final concentration and [STO] the dilution corrected value, similar to the volume correction performed for $F_{i} . F_{o}$ is average $(\mathrm{n}=3)$ fluorescence at the start of the titration, and $F_{\text {final }}$ the average fluorescence and fluorescence quenching after the final substrate addition.

To analyze the fluorescence binding data we applied a 1:1 binding model in accordance with the 1:1 molecular stoichiometry of binding between CaMKK2 and STO-609 as observed in the crystal structure [13].

\section{Dephosphorylation of protein samples}

Dephosphorylation of CaMKK2 and CaMKK2:CaM was achieved by incubating the samples with $0.25 \mu \mathrm{l}$ or $0.5 \mu \mathrm{l}$ lambda phosphatase (Lambda PP; NEB) according to the manufacturers protocol for two hours at $30^{\circ} \mathrm{C}$ prior to measurements.

\section{NanoLC-LTQ Orbitrap Mass Spectrometry}

The purified CaMKK2 and CaMKK2:CaM complex were digested with trypsin (Promega, Madison, WI, USA) and purified using $\mu$-C18 ZipTips (Millipore, Billerica, MA, USA). The tryptic peptides were analyzed using an Ultimate 3000 nano-UHPLC system connected to a Q Exactive mass spectrometer (ThermoElectron, Bremen, Germany). For liquid chromatography separation, an Acclaim PepMap 100 column (C18, $3 \mu \mathrm{m}$ beads, $100 \AA$, $75 \mu \mathrm{m}$ inner diameter) (Dionex, Sunnyvale CA, USA) capillary of $50 \mathrm{~cm}$ bed length was used 
with a flow rate of $300 \mathrm{~nL} / \mathrm{min}$ and a solvent gradient of $7-40 \% \mathrm{~B}$ in $40 \mathrm{~min}$. Solvent $A$ was $0.1 \%$ formic acid and solvent B was $0.1 \%$ formic acid / $90 \%$ acetonitrile. The used mass spectrometer parameter were according to Koehler et al. [16]. Data were acquired using Xcalibur v2.5.5 and raw files were processed to generate peak list in Mascot generic format (".mgf) using ProteoWizard release version 3.0.331. Database searches were performed using Mascot in-house version 2.4.0 to search the SwissProt database (Human, 22.07.2015, 20,204 proteins) assuming the digestion enzyme trypsin, at maximum two missed cleavage sites, fragment ion mass tolerance of $0.1 \mathrm{Da}$, parent ion tolerance of $10 \mathrm{ppm}$ and oxidation of methionines, and acetylation of the protein $\mathrm{N}$-terminus as variable modifications. Scaffold 4.4 (Proteome Software Inc., Portland, OR) was used to validate MS/MS based peptide and protein identifications. 


\section{Results and Discussion}

STO-609 is a small molecule CaMKK2 inhibitor in pre-clinical studies and has been cocrystallized with the kinase domain, reported by Kukimoto-Niino et al. in 2011 (PDB ID: 2ZV2) [13]. Based on the structure of STO-609 we speculated that it could have intrinsic fluorescent properties and during the course of this study this has been confirmed in a recent publication, reporting background fluorescence that interfered with the imaging experiments [17]. To determine the excitation wavelength of STO-609, we varied the excitation wavelength applied to $600 \mathrm{nM}$ free drug in protein buffer by increments of $5 \mathrm{~nm}$ on a range from 200 to $685 \mathrm{~nm}$ measuring an emission wavelengths spectrum from 210 to $700 \mathrm{~nm}$. An excitation wavelength of $400 \mathrm{~nm}$ was defined as optimal with a maximum emission wavelength at $533 \mathrm{~nm}$ (Fig. 1A).

Having established this for the free drug we then sought to assess how this changed upon addition of purified CaMKK2. By excitation at a wavelength of $400 \mathrm{~nm}$, we measured the effect of titrating recombinant CaMKK2 to $2 \mu \mathrm{M}$ STO-609 and collected an emission spectrum in the range from 400 to $750 \mathrm{~nm}$. We observed a $\sim 30 \mathrm{~nm}$ blue-shift with a maximum emission wavelength peak at $\sim 500 \mathrm{~nm}$ (Fig. 1B) accompanied by a 10-fold enhancement of the quantum yield upon saturation (Fig. 1C); two observations that strongly indicate that STO-609 binding to CaMKK2 results in shielding of the drug/chromophore from the aqueous environment, supporting the reported mainly hydrophobic interaction [13]. Therefore, binding of STO-609 to CaMKK2 can be quantitated by the change of intrinsic fluorescence of STO-609 at binding equilibrium, measuring emission at $500 \mathrm{~nm}$. We went on to generate a saturation curve for this fluorescent shift using $210 \mathrm{nM}$ of purified CaMKK2 to which we added increasing concentrations of STO-609 in a range from 0 to $100 \mathrm{nM}$. We achieved saturation at $60 \mathrm{nM}$, taking the fluorescent contribution of STO-609 titrated into buffer in the absence of protein into consideration which we subsequently subtracted from all 
experimental data (Fig. 1D). CaMKK2 is known to exist as a monomer and consequently if all ATP binding pockets are equally accessible we would expect saturation at around $200 \mathrm{nM}$ applying a $1: 1$ binding stoichiometry. To determine the homogeneity of the purification we performed proteomics on the recombinant protein and identified a range of phosphorylation sites, some of which have been previously reported $\left(\mathrm{Ser}^{25}, \mathrm{Thr}^{85}, \mathrm{Thr}^{216}, \mathrm{Thr}^{483}, \mathrm{Ser}^{511}\right.$, and $\left.\operatorname{Thr}^{518}[5,18,19]\right)$ and some of which, particularly those in the activation segment $\left(\mathrm{Ser}^{334}\right.$, $\operatorname{Ser}^{340}, \operatorname{Ser}^{345}, \operatorname{Thr}^{347}, \operatorname{Thr}^{350}$ ), were novel (Fig. 2 and Table S1). To determine whether the phosphorylation status of the kinase impacted on the saturation of the fluorescence signal we incubated the protein with phosphatase prior to measurements. Via mass spectrometry we could determine de-phosphorylation ratios of 0.6 up to 1.0 for the single phosphorylation sites $\mathrm{Ser}^{345}$ and $\mathrm{Thr}^{347}$, which showed prior phosphatase treatment the highest phosphorylation rates. Comparing STO-609 binding affinities for CaMKK2 and CaMKK2:CaM before and after phosphatase treatment, assessed by fluorometric emission as described above, we could see a clear shift of saturation before and after incubation with phosphatase. Phosphatase treatment resulted in saturation of the fluorescence signal for both CaMKK2 and CaMKK2:CaM at higher STO-609 concentrations. Interestingly the impact of phosphatase treatment was to increase the $\mathrm{K}_{\mathrm{D}}$ for CaMKK2 from $\sim 17 \mathrm{nM}$ to $\sim 49 \mathrm{nM}$ whereas the $\mathrm{K}_{\mathrm{D}}$ for CaMKK2:CaM was reduced from $\sim 17 \mathrm{nM}$ to $\sim 7 \mathrm{nM}$. This differential response may imply that there are differential effects of phosphorylation on the STO-609 binding pocket according to whether CaM is also bound to CaMKK2 or not. This merits further molecular dissection in future studies.

Additional impacts on the saturation properties of the assay may arise from protein misfolding or changes in the solubility properties upon the binding of STO-609 independent of the phosphorylation status - these are generic challenges in working with recombinant proteins. 
CaMKK2 has been reported to be regulated by both CaM-binding and by phosphorylation. To assess the impact of CaM interaction on the binding to STO-609 we also co-purified CaMbound CaMKK2 (CaMKK2:CaM) (see DIB report). We applied a 1:1 binding model in accordance with the $1: 1$ molecular stoichiometry of binding observed in the crystal structure of STO-609 bound to the kinase domain of CaMKK2 [13]. In this comparison we observed an identical $\mathrm{K}_{\mathrm{D}}$ of STO-609 binding, $17 \pm 6 \mathrm{nM}$ and $17 \pm 7 \mathrm{nM}$ with and without $\mathrm{CaM}$, respectively, also reflected in overlapping binding curves with and without CaM (Table 1 and Fig. 2C). This also confirmed the robustness and reproducibility of our assay. CaMKK2 is also expressed as a number of isoforms which each have their own regulatory characteristics - differing in their phosphorylation profiles and responses to CaM binding $[3,20]$. In this study we have expressed and purified isoform 3 which, whilst binding to calmodulin, does not show changes in its kinase activity in response to this interaction [3]. The unchanged $K_{D}$ we have observed here with or without calmodulin corroborates this observation but given these isoform studies future work should extend the assay to the full range of isoforms.

This is the first example of a binding study that utilises the intrinsic fluorescent properties of STO-609 to assess and describe the binding properties with its target kinase in a pure in vitro system.

To further verify that the blue-shift in fluorescent upon incubating STO-609 with CaMKK2 was due to its association with the ATP-binding pocket, we titrated STO-609 to $200 \mathrm{nM}$ CaMKK2 up to approximately $50 \%$ saturation ( 60 nm STO-609) before titrating highly concentrated ATP into the sample (Fig. 3A). This experiment allowed us to show the loss of binding between STO-609 and CaMKK2, indicated by a decreasing fluorescent signal at $500 \mathrm{~nm}$, implying its replacement by ATP. However, an ATP / STO-609 ratio of approximately 5000 was needed to lower the signal to $50 \%$ which demonstrates the strong binding affinity between the drug and the kinase. As a control, we added GTP in similar 
amounts. GTP failed to displace STO-609 from the kinase and the potential inner fluorescence effect from purine base present in both ATP and GTP was negligible.

A recent structural study in which STO-609 was soaked into crystals of the kinase domain of CaMKK2 indicated that a number of amino acids in the ATP binding pocket were important for the association [13]. Our fluorescence assay provides the opportunity to further assess the importance of these amino acids for the drug interaction. As a proof-of-principle we mutated Phe ${ }^{267}$, a so-called 'gatekeeper' residue in the ATP-binding pocket, to glycine. This amino acid was selected based on sequence alignments with other kinases that have previously been mutated at 'gatekeeper' residues to support chemical genetic screens for novel kinase substrates in combination with bulky nucleotide analogues [21, 22] (Fig. 3B). This mutation abolished the STO-609 binding to the recombinant kinase in our fluorescent assay which therefore provides a tool for evaluating critical residues directly in an in vitro system (Fig. 3C).

We imagine two different applications for STO-609, based on the presented fluorescent properties. First, it could be used to successfully screen for new inhibitors of CaMKK2 in a competitive or displacement assay on the basis that more effective ATP competitors should out compete STO-609, observed as a drop in fluorescence at $500 \mathrm{~nm}$. Whether this is applicable to other kinases reported to bind STO-609, remains to be investigated. Secondly, STO-609 could be used as a fluorescent chemical probe, as the emission wavelength is in a range (475 to $550 \mathrm{~nm}$ ) that overlaps with the excitation wavelength of yellow fluorescent protein YFP (maximum excitation at $515 \mathrm{~nm}$ ) [23]. This could potentially be used for probing protein-protein interactions with CaMKK2 in live cells, using a fluorescent resonance energy transfer (FRET) assay, equivalent to the classical FRET assay using cyan fluorescent protein (CFP) and YFP [24]. 


\section{Funding sources}

LG and IGM are supported in Oslo by funding from the Norwegian Research Council, Helse Sør-Øst and the University of Oslo through the Centre for Molecular Medicine Norway (NCMM), which is a part of the Nordic EMBL (European Molecular Biology Laboratory) partnership. IGM holds a visiting scientist position with Cancer Research UK through the Cambridge Research Institute and a Senior Honorary Visiting Research Fellowship with Cambridge University through the Department of Oncology. IGM is supported in Belfast by the Belfast-Manchester Movember Centre of Excellence (CE013_2-004), funded in partnership with Prostate Cancer UK. JKJ was supported from the Danish Cancer Society (R56-A2997). PM was supported from the Norwegian Cancer society (project \#4483570). 


\section{References}

[1] S.A. Hawley, D.A. Pan, K.J. Mustard, L. Ross, J. Bain, A.M. Edelman, B.G. Frenguelli, D.G. Hardie, Calmodulin-dependent protein kinase kinase-beta is an alternative upstream kinase for AMP-activated protein kinase, Cell Metab, 2 (2005) 9-19.

[2] A. Woods, K. Dickerson, R. Heath, S.P. Hong, M. Momcilovic, S.R. Johnstone, M. Carlson, D. Carling, Ca2+/calmodulin-dependent protein kinase kinase-beta acts upstream of AMP-activated protein kinase in mammalian cells, Cell Metab, 2 (2005) 21-33.

[3] Y. Ishikawa, H. Tokumitsu, H. Inuzuka, M. Murata-Hori, H. Hosoya, R. Kobayashi, Identification and characterization of novel components of a $\mathrm{Ca2}+$ /calmodulin-dependent protein kinase cascade in HeLa cells, FEBS Lett, 550 (2003) 57-63.

[4] T.R. Soderling, The Ca-calmodulin-dependent protein kinase cascade, Trends Biochem Sci, 24 (1999) 232-236.

[5] M.F. Green, J.W. Scott, R. Steel, J.S. Oakhill, B.E. Kemp, A.R. Means, Ca2+/Calmodulindependent protein kinase kinase beta is regulated by multisite phosphorylation, $\mathrm{J}$ Biol Chem, 286 (2011) 28066-28079.

[6] T. Kitani, S. Okuno, H. Fujisawa, Molecular cloning of $\mathrm{Ca2+/calmodulin-dependent} \mathrm{protein}$ kinase kinase beta, J Biochem, 122 (1997) 243-250.

[7] Y. Nakamura, S. Okuno, T. Kitani, K. Otake, F. Sato, H. Fujisawa, Immunohistochemical localization of $\mathrm{Ca}(2+) / c a l m o d u l i n-d e p e n d e n t$ protein kinase kinase beta in the rat central nervous system, Neurosci Res, 39 (2001) 175-188.

[8] L. Racioppi, P.K. Noeldner, F. Lin, S. Arvai, A.R. Means, Calcium/calmodulin-dependent protein kinase kinase 2 regulates macrophage-mediated inflammatory responses, J Biol Chem, 287 (2012) 11579-11591.

[9] K.A. Anderson, T.J. Ribar, F. Lin, P.K. Noeldner, M.F. Green, M.J. Muehlbauer, L.A. Witters, B.E. Kemp, A.R. Means, Hypothalamic CaMKK2 contributes to the regulation of energy balance, Cell Metab, 7 (2008) 377-388.

[10] M.W. Sleeman, E. Latres, The CAMplexities of central ghrelin, Cell Metab, 7 (2008) 361362.

[11] C.E. Massie, A. Lynch, A. Ramos-Montoya, J. Boren, R. Stark, L. Fazli, A. Warren, H. Scott, B. Madhu, N. Sharma, H. Bon, V. Zecchini, D.M. Smith, G.M. Denicola, N. Mathews, M. Osborne, J. Hadfield, S. Macarthur, B. Adryan, S.K. Lyons, K.M. Brindle, J. Griffiths, M.E. Gleave, P.S. Rennie, D.E. Neal, I.G. Mills, The androgen receptor fuels prostate cancer by regulating central metabolism and biosynthesis, EMBO J, 30 (2011) 2719-2733.

[12] H. Tokumitsu, H. Inuzuka, Y. Ishikawa, M. Ikeda, I. Saji, R. Kobayashi, STO-609, a specific inhibitor of the $\mathrm{Ca}(2+) /$ calmodulin-dependent protein kinase kinase, J Biol Chem, 277 (2002) 15813-15818.

[13] M. Kukimoto-Niino, S. Yoshikawa, T. Takagi, N. Ohsawa, Y. Tomabechi, T. Terada, M. Shirouzu, A. Suzuki, S. Lee, T. Yamauchi, M. Okada-Iwabu, M. Iwabu, T. Kadowaki, Y. Minokoshi, S. Yokoyama, Crystal structure of the $\mathrm{Ca}(2)(+) /$ calmodulin-dependent protein kinase kinase in complex with the inhibitor STO-609, J Biol Chem, 286 (2011) 22570-22579.

[14] R.L. Hurley, K.A. Anderson, J.M. Franzone, B.E. Kemp, A.R. Means, L.A. Witters, The $\mathrm{Ca2}+$ /calmodulin-dependent protein kinase kinases are AMP-activated protein kinase kinases, J Biol Chem, 280 (2005) 29060-29066.

[15] M. Osawa, H. Tokumitsu, M.B. Swindells, H. Kurihara, M. Orita, T. Shibanuma, T. Furuya, M. Ikura, A novel target recognition revealed by calmodulin in complex with $\mathrm{Ca2+-}$ calmodulin-dependent kinase kinase, Nat Struct Biol, 6 (1999) 819-824.

[16] C.J. Koehler, M.O. Arntzen, G.A. de Souza, B. Thiede, An approach for triplex-isobaric peptide termini labeling (triplex-IPTL), Anal Chem, 85 (2013) 2478-2485. 
[17] J.G. Jurcsisn, R.L. Pye, J. Ali, B.L. Barr, C.N. Wyatt, The CamKKbeta Inhibitor STO609 Causes Artefacts in Calcium Imaging and Selectively Inhibits BKCa in Mouse Carotid Body Type I Cells, Adv Exp Med Biol, 860 (2015) 17-24.

[18] H. Tokumitsu, N. Hatano, T. Fujimoto, S. Yurimoto, R. Kobayashi, Generation of autonomous activity of $\mathrm{Ca}(2+) /$ calmodulin-dependent protein kinase kinase beta by autophosphorylation, Biochemistry, 50 (2011) 8193-8201.

[19] H. Daub, J.V. Olsen, M. Bairlein, F. Gnad, F.S. Oppermann, R. Korner, Z. Greff, G. Keri, O. Stemmann, M. Mann, Kinase-selective enrichment enables quantitative phosphoproteomics of the kinome across the cell cycle, Mol Cell, 31 (2008) 438-448.

[20] L.S. Hsu, G.D. Chen, L.S. Lee, C.W. Chi, J.F. Cheng, J.Y. Chen, Human $\mathrm{Ca} 2+/$ calmodulin-dependent protein kinase kinase beta gene encodes multiple isoforms that display distinct kinase activity, J Biol Chem, 276 (2001) 31113-31123.

[21] K. Shah, K.M. Shokat, A chemical genetic approach for the identification of direct substrates of protein kinases, Methods Mol Biol, 233 (2003) 253-271.

[22] H. Tokumitsu, M. Muramatsu, M. Ikura, R. Kobayashi, Regulatory mechanism of Ca2+/calmodulin-dependent protein kinase kinase, J Biol Chem, 275 (2000) 20090-20095.

[23] T. Nagai, K. Ibata, E.S. Park, M. Kubota, K. Mikoshiba, A. Miyawaki, A variant of yellow fluorescent protein with fast and efficient maturation for cell-biological applications, Nat Biotechnol, 20 (2002) 87-90.

[24] J. Zhang, R.E. Campbell, A.Y. Ting, R.Y. Tsien, Creating new fluorescent probes for cell biology, Nat Rev Mol Cell Bio, 3 (2002) 906-918. 


\section{Figure/Table Legends}

Figure 1. Fluorometric assay development. (A) Determining the intrinsic fluorescence of STO-609 by a 3D spectrum measurement. The maximum excitation wavelength is $400 \mathrm{~nm}$, the maximum emission wavelength is $533 \mathrm{~nm}$. (B) At an excitation wavelength of $400 \mathrm{~nm}$, titration of recombinant CaMKK2 to $2 \mu \mathrm{M}$ STO-609 caused a blue-shift of emission wavelength from 533 towards $500 \mathrm{~nm}$. (C) Upon saturation of the STO-609 signal by increasing concentrations of CaMKK2 to 200 nM STO-609, we obtained a ten-fold increase of the signal. (C, insert) Normalized fluorescence intensity ( $F / F$ total) measured at 400/500 nm plotted against CaMKK2 concentration. (D) Normalized fluorescence intensity (F/F total) measured at 400/500 nm for increasing STO-609 concentrations in protein buffer, with (blue) and without CaMKK2 (purple). Saturation in fluorescent levels of the drug was reached at STO-609 concentrations equivalent to approximately $1 / 4$ times the concentration of protein present and continued linearly as indicated, parallel to the intrinsic fluorescence of STO-609 itself. (D, insert) Intrinsic fluorescence signal of STO-609 remained linear above $800 \mathrm{~nm}$, indicating negligible contribution from inner fluorescence. Error bars represent the standard deviation from three independent experiments.

Figure 2. Phosphorylation studies. (A) Mass spectrometry analyses of CaMKK2 phosphorylation, highlighting phosphorylation sites detected in the kinase domain (KD), purple, of CaMKK2. A cluster of novel phosphorylation sites corresponds to the location of the activation segment (AS), green. RP: arginine-proline-rich site; AID: autoinhibitory domain. (B) Cartoon representation of the CaMKK2 KD structure (PDB ID: 2ZV2) [13]. Phosphorylated Ser/Thr are labelled and highlighted as spheres. The activation segment from beta strand $8(\beta 8)$ to alpha-helix EF $(\alpha E F)$ is coloured green and the Arg-Pro-rich insert (RP-insert) drawn in grey, that includes a single phosphorylated $\mathrm{Thr}^{216}$. STO-609 is shown as purple sticks. (C) Fluorometric binding assay of STO-609 to $210 \mathrm{nM}$ CaMKK2 (purple) or 
CaMKK2:CaM (blue) before (O) and after $(\mathbf{\Delta})$ incubation with Lambda PP. Higher STO-609 concentrations were needed to saturate CaMKK2 after incubation with Lambda PP. Values represent means of three independent experiments; error bars represent the standard deviation.

Figure 3. (A) ATP replacement assay. ATP titrated to STO-609-bound CaMKK2 releases STO-609. Four individual measurements taken for ATP replacement assay (green and blue shades). GTP titration (purple) to STO-609-bound CaMKK2 shows no effect on fluorescent levels of CaMKK2-bound STO-609. Representative experiments and corresponding fits are shown. (B) Sequence alignments of CaMKK2 with CaMKK1 and CDK2. This highlights the conserved gatekeeper residue (purple box) in CaMKK2, Phe ${ }^{267}$, that corresponds to CaMKK1 $\mathrm{Phe}^{230}$ and CDK2 Phe ${ }^{80}$. (C) Increasing concentrations of STO-609 were added to $210 \mathrm{nM}$ CaMKK2 (purple) or CaMKK2 $\mathrm{F}^{267} \mathrm{G}$ (pink), respectively. All curves represent an average of three independent experiments to give a qualitative view of the binding assay and its reproducibility.

Table 1. $K_{D}$ values determined by fluorometric binding assay. Affinity measurements of STO609 binding to CaMKK2 based on perturbation of intrinsic STO-609 fluorescence experiments performed in the absence or presence of CaM and with and without CaMKK2 treatment with Lambda PP.

CaMKK2 binding activity: $\quad[\text { CaMKK2 }]_{\text {calculated }} /[\text { CaMKK2 }]_{\text {theoretical }} \quad{ }^{*} 100 \%$ 
Tables

$\underline{\text { Table } 1}$

CaMKK2 binding activity

CaMKK2

CaMKK2:CaM
$8 \%$

$10 \%$
$\mathrm{K}_{\mathrm{D}}$

$17 \pm 7 \mathrm{nM}$

$17 \pm 6 \mathrm{nM}$
$K_{D}$ (+ Lambda PP)

$49 \pm 6 \mathrm{nM}$

$7.4 \pm 0.6 \mathrm{nM}$ 
A

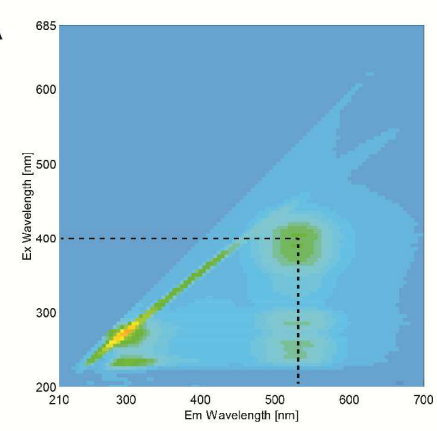

C

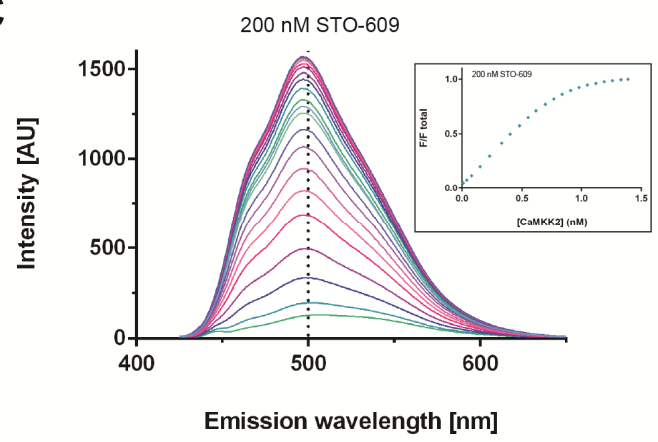

B

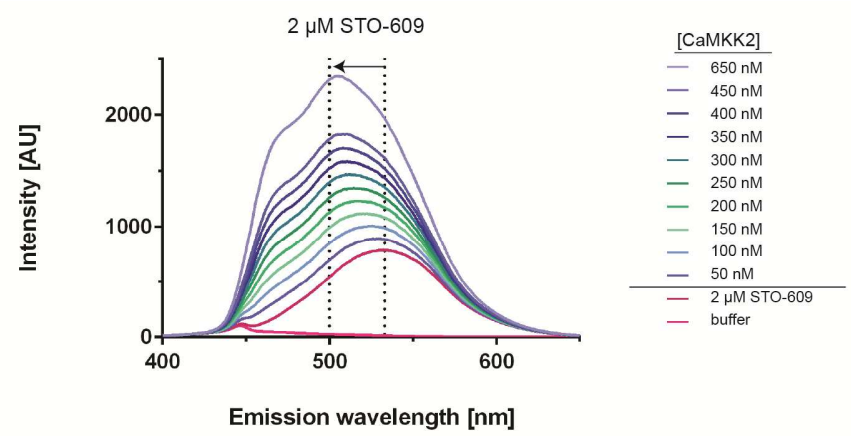

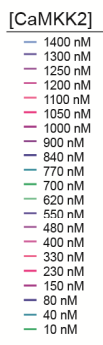

D

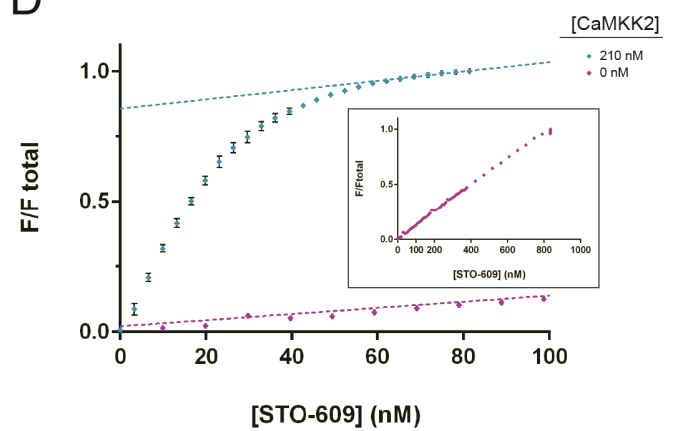


A

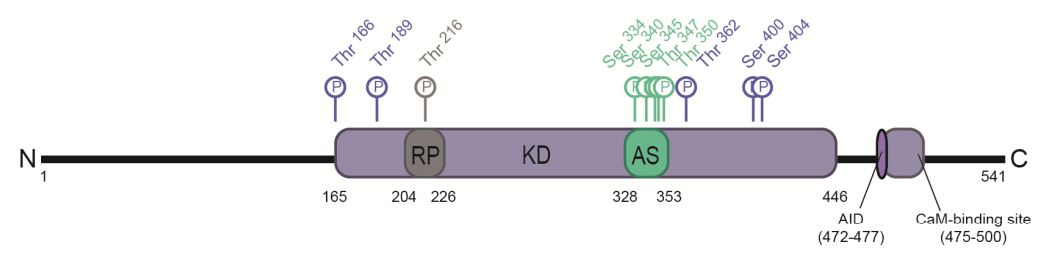

B

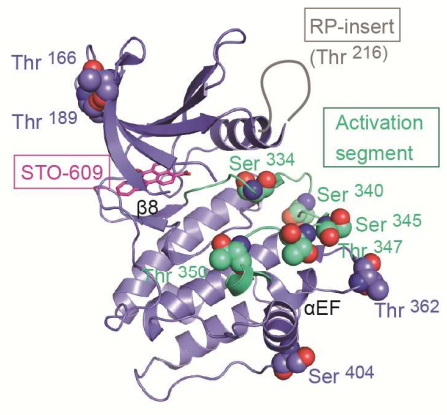

C

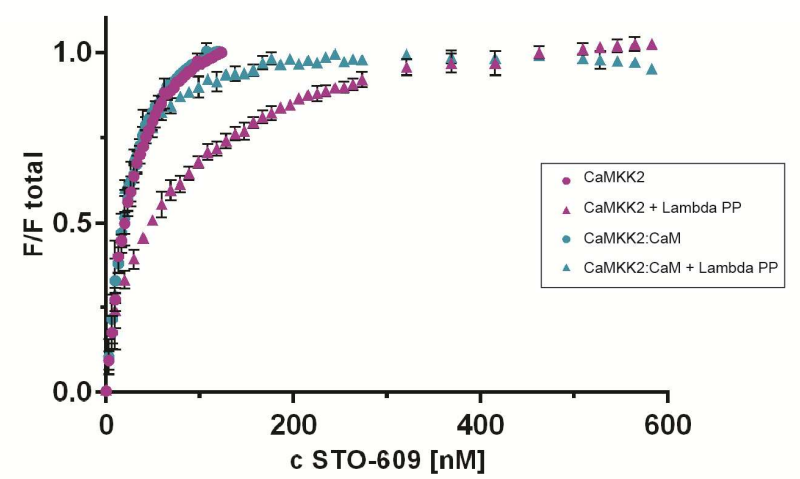


A

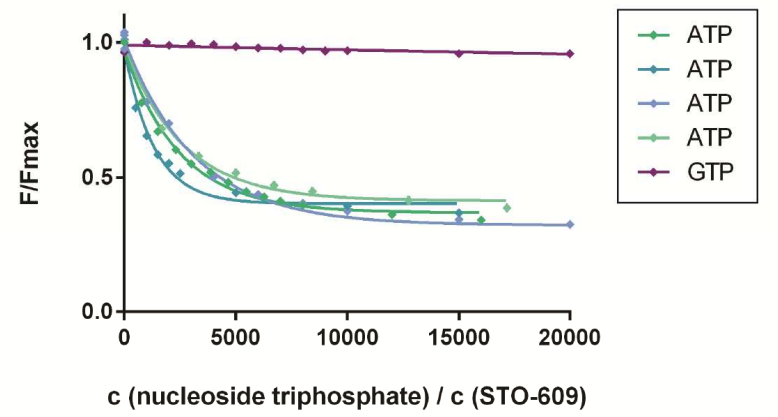

C

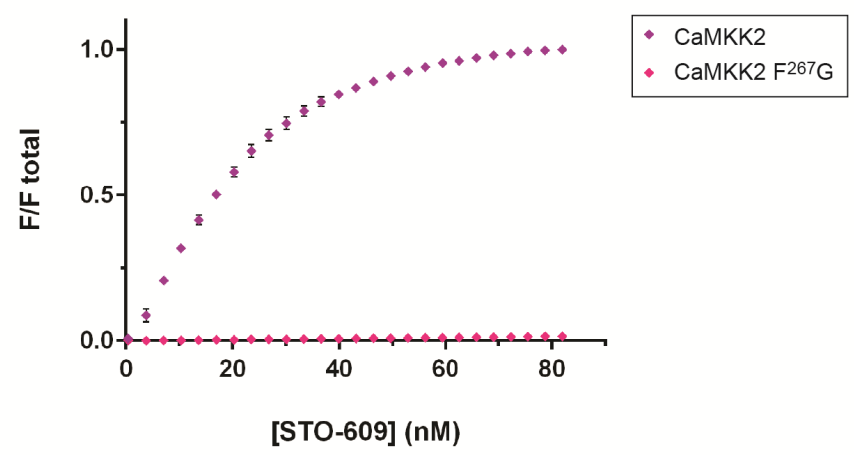

[STO-609] (nM)
B

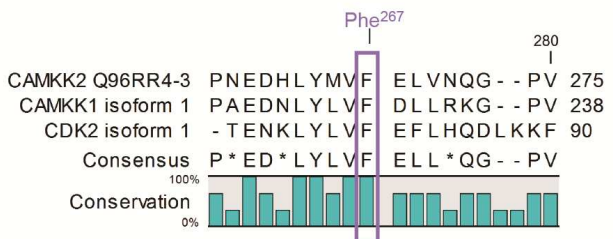




\section{Highlights}

- STO-609 is intrinsically fluorescent and is used to assess binding by CAMKK2

- Phenylalanine-267 is critical for the interaction with STO-609

- Recombinant CAMKK2 is phosphorylated and we find novel activation segment sites

- Binding of STO-609 by recombinant CaMKK2 is phosphorylation-dependent

- The assay provides a tool to support future drug screening and functional studies 